\title{
Attentional blink and attentional capture: Endogenous versus exogenous control over paying attention to two important events in close succession
}

\author{
THOMAS M. SPALEK, LAURA J. FALCON, and VINCENT DI LOLLO \\ Simon Fraser University, Burnaby, British Columbia, Canada
}

\begin{abstract}
Identification of the second of two targets is impaired if it is presented less than about 500 msec after the first. This attentional blink (AB) occurs under dual-task conditions in which observers are required to report both targets. $\mathrm{AB}$ magnitude has been estimated by subtracting the accuracy scores in the dual task from the corresponding scores in a single task in which observers are instructed to ignore the first target. Experiment 1 showed this procedure to be inappropriate, because the first target cannot be ignored. The remaining three experiments elaborated on this finding and revealed separate endogenous and exogenous sources of the second-target deficit. A parallel was drawn between the $\mathrm{AB}$ deficit and the deficit observed in attentional capture. Both types of deficit can be explained on the basis of a hybrid input-filtering model in which endogenous and exogenous factors are subserved by different pathways.
\end{abstract}

There is a cost to attending to two visual targets presented in rapid sequence. Identification accuracy is high for the first target but is impaired for the second. This second-target deficit, known as the attentional blink (AB), has been studied with a paradigm called rapid serial visual presentation (RSVP), in which the two targets are inserted in a stream of distractors (see, e.g., Chun \& Potter, 1995; Raymond, Shapiro, \& Arnell, 1992). All items in the RSVP stream are presented at the same spatial location, at a rate of approximately $10 \mathrm{items} / \mathrm{sec}$. The temporal lag between the two targets is manipulated by varying the number of intervening distractors. The second-target deficit is most pronounced at short intertarget lags, with performance recovering progressively as the lag is increased.

Theoretical accounts of the $\mathrm{AB}$ have focused on the processing of the first target as the prime determinant of the second-target deficit. In these accounts (e.g., Chun \& Potter, 1995; Jolicœur \& Dell'Acqua, 1998; Raymond et al., 1992), the requirement to attend to the first target is said to delay the allocation of attentional resources to the second target for a period of several hundred milliseconds. As a result, if the second target is presented soon after the first, it cannot be processed immediately, and is vulnerable to decay or overwriting by subsequent stimuli

This work was sponsored by grants from Simon Fraser University (President Research Grant and Endowed Research Fellowship) to T.M.S. and from the Natural Sciences and Engineering Research Council of Canada to V.D.L. We acknowledge the expert assistance of Shannon Gaudry in the collection of the data. Correspondence concerning this article should be addressed to T. M. Spalek, Department of Psychology, Simon Fraser University, 8888 University Drive, Burnaby, BC, V5A 1S6 Canada (e-mail: tspalek@sfu.ca). (see, e.g., Giesbrecht \& Di Lollo, 1998). As the intertarget interval is increased, processing of the first target is more likely to be completed so that attentional resources are again available when the second target arrives.

A commonly used control condition for assessing the effect of first-target processing on the second-target deficit is to instruct the observers to ignore the first target (Arnell \& Jolicœur, 1999; Christmann \& Leuthold, 2004; Chun, 1997; Raymond et al., 1992; Seiffert \& Di Lollo, 1997; Shapiro, Raymond, \& Arnell, 1994; Ward, Duncan, \& Shapiro, 1997). This control condition is based on the assumption that when the first target is ignored, all attentional resources are available for processing the second target. This rationale leads to the expectation that second-target accuracy in the control condition should remain constant throughout the domain - namely, it should not vary as a function of the temporal lag between the two targets.

This is precisely what was found by Raymond et al. (1992) in the original demonstration of the AB. In other studies, however, such a control condition yielded secondtarget deficits that could be as large as those obtained in the corresponding experimental conditions in which observers reported both targets (see, e.g., Christmann \& Leuthold, 2004; Chun, 1997). The main objective of the present work was to investigate the factors underlying the inconsistent pattern of second-target deficit obtained in this control condition.

The conceptual framework for the present research was provided by an attentional phenomenon known as contingent capture that is observed in studies in which a target must be found among distractors. In such studies, a distractor captures attention when it shares the target's defining characteristic, resulting in slower identification 
of the target (Folk, Remington, \& Johnston, 1992). In the experimental literature, this delay in target identification has been attributed exclusively to the time it takes to make an involuntary shift in the focus of attention to a distractor location. It has recently been shown, however, that such a delay can also arise from the time it takes to process the distractor item, even in the absence of a concomitant spatial shift in the focus of attention (Ghorashi, Zuvic, Visser, \& Di Lollo, 2003). It has also been shown that the performance deficit associated with contingent capture is obtained not only when the response measure is speed of responding but also when it is accuracy of target identification (Egeth, Folk, Leber, Nakama, \& Hendel, 2001; Ghorashi et al., 2003).

A close parallel can be drawn between the targetidentification deficit obtained in contingent capture and the one obtained in the conventional control condition in AB experiments. In both cases, observers must identify a single target, and are instructed to ignore all stimuli other than the target. In the case of contingent capture, the deficit occurs when the distractor shares the defining characteristic of the target. We suggest that this may also be the case for the conventional control condition in $\mathrm{AB}$ studies.

There is no question that the $A B$ deficit vanishes or is much reduced when the requirement to process the first target is removed. However, the literature indicates this to be true only when the first target does not possess the distinguishing characteristic of the second target. For example, in the study of Raymond et al. (1992), the first target was a white letter to be identified and the second a black $\mathrm{X}$ to be detected. Thus, the two targets differed distinctly from one another both in defining characteristic and in the type of response required. The same was true in the study of Seiffert and Di Lollo (1997), in which the first target was a bright letter to be identified and the second a dim $\mathrm{X}$ to be detected. In these experiments, the first target did not possess the distinguishing characteristic of the second target and, therefore, the two targets could be differentiated easily. As a consequence, contingent capture did not occur, and the $\mathrm{AB}$ deficit was eliminated or much reduced.

In contrast, when the two targets share the same defining characteristic, instructions to ignore the first target are ineffectual. Not only is a second-target deficit obtained reliably, but its magnitude is almost as large as in the corresponding experimental condition in which observers are required to process the first target. This was first reported by Chun (1997) in a study in which both targets were letters to be identified. Similar results have been reported by Christmann and Leuthold (2004) and by Maki, Bussard, Lopez, and Digby (2003), who also used two letter targets. This strongly suggests that the first target is hard to ignore when it shares a distinguishing characteristic with the second target. In light of these results, a parallel between the contingent capture paradigm and the conventional control condition in the $\mathrm{AB}$ paradigm becomes compelling. In both paradigms, a to-be-ignored leading item, whether first target or distractor, will interfere with the perception of a trailing target only when the two have the same defining characteristic.

\section{The Subtraction Procedure of Estimating the AB Deficit}

It follows from these considerations that the conventional control procedure in AB experiments may be appropriate only when the first target does not possess the defining characteristic of the second target. That control procedure becomes inappropriate, however, when the first target possesses the defining characteristic of the second. In this case, the procedure is inappropriate because the control condition itself produces a second-target deficit. This problem is most evident when the magnitude of the $\mathrm{AB}$ deficit is estimated by subtracting the score at each lag in an experimental (dual-task) condition from the corresponding score in a control (single-task) condition in which the first target is to be ignored. For example, suppose that in a hypothetical experiment, such a subtraction procedure were to yield no $\mathrm{AB}$ deficit. The question that needs to be asked is: Was the $\mathrm{AB}$ absent because there was no lag-related deficit in the experimental condition, or was it absent because the control condition exhibited a deficit of a magnitude roughly comparable to that exhibited by the experimental condition? If the control condition itself revealed a lag-related deficit, subtracting two sets of comparable scores would result in a flat difference function across lags. But this would not mean that the $A B$ was absent. Rather, it would mean that $A B$ deficits of approximately equal magnitudes were obtained in the experimental and in the control conditions.

But this problem is not limited to the issue of whether the $\mathrm{AB}$ is present or absent. The subtraction (control minus experimental) procedure can potentially distort the evidence relating to the rate at which the $\mathrm{AB}$ develops. A case in point can be found in a study by Kellie and Shapiro (2004), especially in Experiment 2, Figures 6A and $6 \mathrm{~B}$. Figure $6 \mathrm{~A}$ illustrates $\mathrm{AB}$ magnitude estimated by the subtraction procedure described above. The time course of the AB illustrated in Figure 6A followed an inverted-U-shaped function showing a total absence of $\mathrm{AB}$ at the shortest lag, a gradual increase to a maximum at intermediate lags, and a return to zero at the longest lag. This U-shaped function could represent the genuine course of the AB deficit, but it could also be an artifact of the subtraction procedure. Suppose that the control condition produced an initially strong but short-lived AB, and the experimental condition produced a longer-lasting $\mathrm{AB}$. Subtracting the latter from the former would produce the performance curve illustrated in Figure 6A of Kellie and Shapiro.

That this was indeed the case in Experiment 2 of Kellie and Shapiro (2004) is confirmed by the data in their Figure 6B, which shows separate performance curves for the experimental and the control condition. An asymptotic level of approximately $88 \%$ correct identifications of the second target can be estimated by averaging the performance of the control group over the five longest lags, at which performance no longer showed systematic improvement. In relation to this estimate, it can be seen that an $\mathrm{AB}$ deficit of approximately $12 \%$ was exhibited by both the experimental and the control conditions at the shortest lag. 
Thus, the control condition revealed an initial $\mathrm{AB}$ deficit that was just as large as that in the experimental condition. Therefore, the absence of an $\mathrm{AB}$ at the shortest lag in Figure 6A must be attributed not to a genuine absence of a second-target deficit in the experimental condition but to the inappropriate use of the subtraction procedure. Interpretation of the inverted-U-shaped temporal course of the AB illustrated in Kellie and Shapiro's Figure 6A is beset by similar considerations.

Comparing an experimental condition with a control condition in which the first target is to be ignored is a relatively common practice in the $\mathrm{AB}$ literature (see, e.g., Christmann \& Leuthold, 2004; Jolicœur \& Dell'Acqua, 1998; Seiffert \& Di Lollo, 1997). To be sure, in these studies, the experimental function was not actually subtracted from the control function, as in the Kellie and Shapiro (2004) study, but such a subtraction was implied in the comparison between the two functions.

\section{Objectives of the Present Work}

Although the evidence in the literature is suggestive, a systematic investigation of the consequences of using different control procedures remains to be done. This was one of the two main objectives of the present study. A second objective was to pursue the parallel between the target-identification deficit obtained in the conventional control condition in the $\mathrm{AB}$ and in the contingent capture paradigms.

In all present experiments, the RSVP stream contained two items designated as targets. However, all conditions, except for the dual conditions in Experiments 1 and 3, were single-task conditions in which observers were instructed to ignore the first target, when present, and report only the second. In these single-task conditions, therefore, the to-be-ignored first target was functionally equivalent to the other distractors in the RSVP stream. Its main function was to act as a reference point in the RSVP stream from which to time the temporal lag until the presentation of the second target. This means that the first target was presented in the sequential RSVP position conventionally known as lag 0 . For consistency of exposition, however, we refer to the lag 0 item as the "first target," even though the observers are required to ignore it. Similarly, we refer to the other target in the RSVP stream as the "second target" even though it was the only target to be reported.

\section{EXPERIMENT 1}

We have argued that the conventional single-task control procedure in which the observer is required to ignore the first target can yield misleading results. This is because the mere act of ignoring the first target involves a degree of processing that demonstrably interferes with processing of the second target. In this case, processing of the second target will occur under what are effectively dual-task conditions.

If the second-target deficit is to be attributed unambiguously to the consequences of processing the first target, there is another factor that needs to be controlled: the sim- ilarity between the second target and the distractors in the RSVP stream. It has been shown that when the items in the distractor stream are highly similar to the second target, a large second-target deficit occurs that is independent of first-target processing, because it occurs even at very long intertarget lags (Visser, Bischof, \& Di Lollo, 2004). In this case, the appropriate control condition is one in which the similarity between the distractors and the second target is maintained, but the first target is replaced with a distractor. In other words, the experimental and the control conditions would differ only with respect to the requirement to process the first target. Two sources of confounding would be factored out by such a control condition: the processing requirement of ignoring the first target, and the effect of target-distractor similarity. The magnitude of the $A B$ deficit attributable to the effects of processing the first target can then be estimated by subtracting the level of second-target performance in the dual-task condition from that in the single-task condition.

Experiment 1 was designed to perform a direct comparison between two types of single-task control conditions. One was the conventional single-task control, in which observers were required to ignore the first of two targets in the RSVP stream. In the other, the first target was replaced by a distractor in the RSVP stream. We reasoned that a comparison between the levels of second-target performance in the two single-task conditions would reveal the cost associated with the requirement to ignore the first target with target-distractor similarity held constant. A conventional dual-task condition was included to provide a baseline against which any second-target deficit in the two control conditions could be compared.

\section{Method}

Participants. Thirty-six undergraduate volunteers participated for class credit or payment. All reported normal or corrected-tonormal vision and were naive to the purpose of the experiment. They were assigned randomly to one of three groups, each with 12 participants.

Apparatus and Stimuli. The stimuli consisted of sequences of digits (0-9) and letters (all uppercase letters of the English alphabet, except I, O, Q, and Z) presented in RSVP in the center of a computer monitor. All stimuli were white, subtended approximately $1^{\circ}$ of visual angle, and were presented on a black background (approximately $0.5 \mathrm{~cd} / \mathrm{m}^{2}$ ) at a luminance of $50 \mathrm{~cd} / \mathrm{m}^{2}$, as measured by a Minolta LS 100 luminance meter. Observers sat in a darkened room, and viewed the displays from a distance of approximately $60 \mathrm{~cm}$.

Procedure. Experiment 1 comprised three conditions with 12 participants in each: the dual condition, the single-ignore condition, and the single-absent condition. The procedural details common to all conditions were as follows. At the beginning of a session, observers were required to read the instructions displayed on the screen, and were invited to ask questions to clarify procedural points. At the beginning of each trial, a small fixation cross was presented in the center of the screen, indicating the location at which the RSVP stream of digits and letters was about to appear. Participants initiated each trial by pressing the space bar. An RSVP stream of digits and letters was displayed directly afterward. Each item in the RSVP stream remained on the screen for $100 \mathrm{msec}$, and was replaced immediately by the next item in the stream, yielding a presentation rate of 10 items/sec.

In the dual condition, the RSVP stream contained a variable number of digit distractors and two letter targets, selected randomly, 
without replacement, from all letters of the English alphabet. The number of distractors preceding the first target was determined randomly on each trial and varied between 11 and 20. On any given trial, the distractors were selected randomly, with replacement, from the set of digits $0-9$, with the constraint that the selected digit was not one of the two preceding items. The second target was then presented at one of four lags after the first target: 100, 200,300, or $700 \mathrm{msec}$. Digit distractors continued to be presented during the intertarget interval. The second target was then presented, and was followed by one digit distractor that acted as a mask. Participants were required to ignore the distractors and to report the identity of the two targets by pressing the corresponding keys on the keyboard.

The single-ignore condition was the same as the dual condition except that the participants were instructed to ignore the first target and report the identity of only the second target. The single-absent condition was the same as the single-ignore condition except that the first target was replaced by a digit distractor.

In each of the three conditions, observers were given 20 practice trials at the beginning of the session. These were followed by a total of 200 trials, 50 for each of the four intertarget lags.

\section{Results and Discussion}

Two methods of scoring the responses. The percentages of correct responses for the second target, averaged over participants, are illustrated in Figure 1. The results of the dual condition are presented in two different ways, depending on the method of scoring: ordered or not ordered. It is a scoring convention in $\mathrm{AB}$ experiments to ignore the order in which the two targets are reported. For example, if the first target is the letter $\mathrm{G}$, the response is scored as correct if either of the two reported letters is a G. We refer to this as not-ordered scoring. This method of scoring is not possible in the single-ignore condition of the present experiment because observers reported only the second target. This means that if an observer confused the order of presentation of the two targets and reported the first target instead of the second, the response was scored as incorrect. This method of scoring is inherently ordered because it is predicated on the perception of the two targets in the correct order.

For this reason, the results of the dual condition were scored in two ways: one in which the order of responding was taken in consideration (ordered scoring; filled circles with solid lines in Figure 1) and the other in which the order of responding was not taken in consideration (notordered scoring; filled circles with dotted lines in Figure 1). It is clear in Figure 1 that the not-ordered method confers an advantage at the shorter lags, when reversals are more likely to occur because the two targets are presented in close temporal contiguity. That advantage is reduced and eventually disappears as the lag is increased, because of the greater ease of separating the two targets in time. The present analyses were based exclusively on data obtained using the ordered scoring method.

Analysis of results. The results were analyzed in a 3 (condition: single absent, single ignore, and dual [ordered]) $\times 4$ (lag: 100, 200, 300, and $700 \mathrm{msec}$ ) ANOVA. The analysis revealed significant effects of condition $\left[F(2,33)=53.82, M S_{\mathrm{e}}=301.34, p<.001\right]$ and lag $\left[F(3,99)=99.92, M S_{\mathrm{e}}=57.94, p<.001\right]$ and a significant interaction $\left[F(6,99)=30.06, M S_{\mathrm{e}}=57.94, p<\right.$

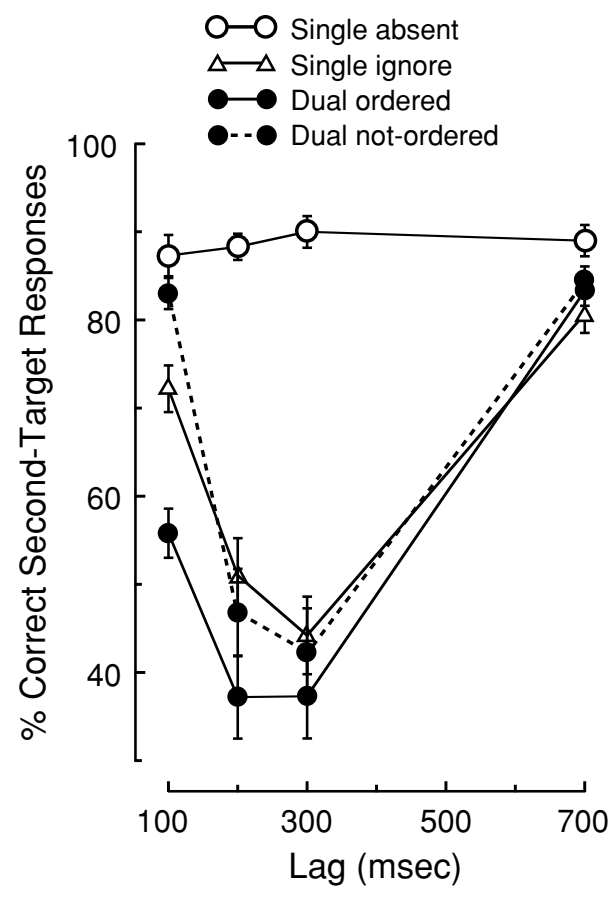

Figure 1. Mean percentages of correct responses in Experiment 1. In the dual condition, participants identified two letters inserted in a stream of digit distractors. The dual not-ordered function represents mean percentages of correct second-target responses, regardless of order of report-namely, whether the second target was reported as the first or the second of two targets. The dual-ordered function represents mean percentages of correct second-target responses, given that it was reported in the correct order-namely, as the second of the two targets. In the single-ignore condition, observers were instructed to ignore the first target and identify only the second target. In the singleabsent condition, the stream of distractors contained only one target, corresponding to the second target in the other two conditions. Error bars represent one standard error of the mean.

.001]. All conditions illustrated in Figure 1, except for the single-absent condition, exhibit a U-shaped trend over lags, with the deficit being most pronounced at a lag longer than $100 \mathrm{msec}$. The virtually unimpaired performance at the shortest lag has been referred to as lag-1 sparing (Potter, Chun, Banks, \& Muckenhoupt, 1998).

"Ignore" and "absent" control conditions. A notable result in Figure 1 is the pronounced $\mathrm{AB}$ deficit obtained in the single-ignore condition. It must be emphasized that this is the control condition typically used in AB experiments. The lag-dependent deficit can be understood on the assumption that the very act of ignoring the first target involves the critical first step of realizing that the stimulus is indeed a target. This first step obviously involves at least some processing of the first target, with consequent impairment in the processing of the second target. Clearly, such a control condition falls short of meeting the objective of assessing second-target performance unencumbered by the requirement to process the first target.

This finding has direct implications for the practice of estimating the magnitude of the $\mathrm{AB}$ deficit by subtracting 
the level of second-target performance in the dual-task condition from that of the single-task control (see, e.g., Kellie \& Shapiro, 2004). Such a subtraction procedure would result in an underestimation of the magnitude of the $\mathrm{AB}$ deficit, to the extent that the control condition itself produces a second-target deficit, as was the case in the present experiment. Indeed, if the dual (not-ordered) function in Figure 1 were to be subtracted from the singleignore function, then hardly any second-target deficit would be in evidence. With reference to Figure 1, it is clear that no such underestimation would occur from a subtraction procedure involving the dual and the singleabsent conditions.

An input-filtering account. A simple account of the second-target deficit obtained in the single-ignore condition can be developed on the basis of the input-filtering model proposed by Visser, Bischof, and Di Lollo (1999; Ghorashi et al., 2003). In that model, processing is said to occur in two broadly sequential stages. The first is a highcapacity, parallel-processing stage, whose main functions are to detect potential targets and to encode them in readiness for further processing. This initial stage is followed by a capacity-limited serial stage, in which stimuli are consolidated and encoded in a form suitable for subsequent report.

The basic tenets of this model bear distinct similarities to other two-stage models, such as those proposed by Broadbent and Broadbent (1987), Chun and Potter (1995), Di Lollo (1980), Duncan (1980), and Hoffman (1979). What distinguishes the model of Visser et al. (1999) from these earlier two-stage models is the explicit filtering function assigned to the first processing stage. Initial processing is said to be performed by input-filtering mechanisms whose functional characteristics are programmable under the control of higher brain regions.

Programming the input filter is said to be part of a goaldirected process aimed at tuning the visual system to those attributes and characteristics of incoming stimuli that are likely to prove useful for performing the task at hand. When a task involves searching for a target among distractors, the filter is said to be optimally tuned to the defining characteristic of the target. The better the fit of an incoming stimulus to the filter setting, the greater the probability that the stimulus will be tagged as a potential target, pass the filter, and gain access to the second processing stage. It is assumed that, in the first processing stage, all stimuli, whether tagged as potential targets or not, are subject to decay and masking by trailing stimuli.

Interpretation of the present results in terms of this twostage model is straightforward. In the single-ignore condition, the input filter was set to pass letter-like stimuli, because that was the defining characteristic of the to-bereported second target. Since the to-be-ignored first target was also a letter, it matched the filter setting and thus had a relatively high probability of gaining access to the second processing stage. A second-target deficit then followed, because, in this model, processing at the second stage is strictly serial: Only one item can be processed at a time. Thus, if the second target arrives while the second stage is busy, it is delayed in the first stage even if it matches the filter's characteristics. During this delay, the second target can be overwritten before gaining access to the second stage and, as a consequence, will fail to be identified. Within this conceptual framework, the second-target deficit obtained in the single-ignore condition belongs to the same class of events as that obtained in the dual condition, and is explained in the same terms.

\section{EXPERIMENT 2}

Experiment 2 was designed to examine the hypothesis that the results obtained in the dual condition and in the single-ignore (conventional control) condition in Experiment 1 are based on common underlying mechanisms. According to this hypothesis, factors that influence performance in the dual condition should have corresponding effects on the single-ignore condition. For example, it is known that the magnitude of the second-target deficit in conventional dual-task $\mathrm{AB}$ experiments is related directly to the similarity between targets and distractors. This is true whether the similarity is structural (Chun \& Potter, 1995; Visser et al., 2004), semantic (Maki, Couture, Frigen, \& Lien, 1997), or conceptual (Dux \& Coltheart, 2005). According to the hypothesis of common underlying mechanisms, increasing target-distractor similarity should increase the second-target deficit in the singleignore condition as well.

This hypothesis was tested in Experiment 2 by varying the degree of similarity between the second target and the notional first target. In every case, the second target was the only item to be reported and, therefore, was presumed to determine the setting of the input filter. The degree of similarity was manipulated by varying the number of attributes that the to-be-ignored first target shared with the second target. The similarity between the second target and the distractors was held constant throughout. There were three conditions: low similarity, medium similarity, and high similarity. In all three conditions, the second target was a red letter and the distractors were white digits. In the low-similarity condition, one of the white digit distractors was denoted as the "first target," and was presented at specified lags before the second target. This was a dummy variable that enabled comparison across lags with the other conditions. Thus, in the low-similarity condition, the first target shared only one relevant attribute - structural features - with the second target.

The medium-similarity condition was the same as the low-similarity condition except that the digit denoted as the "first target" was colored red. In this condition, the first target shared two salient attributes with the second target: structural features and color. The high-similarity condition was the same as the medium-similarity condition except that the first target was a red letter. In this condition, the first target shared three attributes with the second target: structural features, color, and category.

It must be emphasized that in all three conditions, the first target played the role of a distractor. To the extent that the observers could follow instructions to ignore all items 
other than the second target, the similarity manipulation should have no effect. On the other hand, if performance in the control condition is based on the same mechanisms as in the conventional dual-task condition, identification accuracy should be lowest in the high-similarity condition, in which the first target matches the filter setting most closely, and highest in the low-similarity condition, in which the match is poor.

\section{Method}

Apparatus, stimuli, and procedures in Experiment 2 were the same as in Experiment 1, except for the following. Forty-two observers were assigned randomly to one of three conditions of 14 observers each: high similarity, medium similarity, and low similarity. In all three conditions, the distractors were white digits. In the high-similarity condition, the two targets were red letters, and the participants were required to ignore the first letter and identify only the second. The medium-similarity condition was the same as the high-similarity condition except that the to-be-ignored first target was a red digit. The low-similarity condition was the same as the medium-similarity condition except that the first target was replaced by a white digit distractor, and the observers were required to report the only letter in the RSVP stream.

\section{Results and Discussion}

The mean results for all three conditions are illustrated by the solid-line functions in Figure 2. The results of the single-ignore condition from Experiment 1 have been added to Figure 2 (dotted-line function) for ease of comparison. The results of Experiment 2 were analyzed in a 3 (condition: high, medium, and low similarity) $\times 4$ (lag: 100, 200, 300, and $700 \mathrm{msec}$ ) ANOVA. The analysis revealed significant effects of condition $[F(2,39)=$ $\left.34.28, M S_{\mathrm{e}}=243.58, p<.001\right]$ and lag $[F(3,117)=$ 23.51, $\left.M S_{\mathrm{e}}=55.63, p<.001\right]$ and a significant interaction $\left[F(6,117)=11.58, M S_{\mathrm{e}}=55.63, p<.001\right]$. The significant effect of condition reflects the progressive improvement in second-target identification as the similarity with the first target was decreased. The significant interaction reflects differences in the temporal course of the second-target deficit over lags in the three conditions. Subsidiary ANOVAs confirmed that the effect of lag was significant in the high-similarity and medium-similarity conditions $\left[F(3,39)=18.63, M S_{\mathrm{e}}=127.18, p<.001\right.$, and $F(3,39)=6.59, M S_{\mathrm{e}}=31.95, p=.001$, respectively $]$ but not in the low-similarity condition $[F(3,39)=2.18$, $\left.M S_{\mathrm{e}}=7.77, p=.11\right]$.

Effect of target-distractor similarity. As noted above, the magnitude of the second-target deficit in conventional dual-task $\mathrm{AB}$ studies is known to be directly related to the similarity between targets and distractors (Chun \& Potter, 1995; Dux \& Coltheart, 2005; Maki et al., 1997; Visser et al., 2004). The results of the present experiment show this to also be true for the conventional control condition in which the first target is to be ignored. The magnitude of the second-target deficit, therefore, is affected in similar ways when the first target is to be ignored (i.e., plays the role of a distractor) and when it is to be reported. This is consistent with the hypothesis that the second-target deficit obtained in $\mathrm{AB}$ experiments and in the conventional control condi-

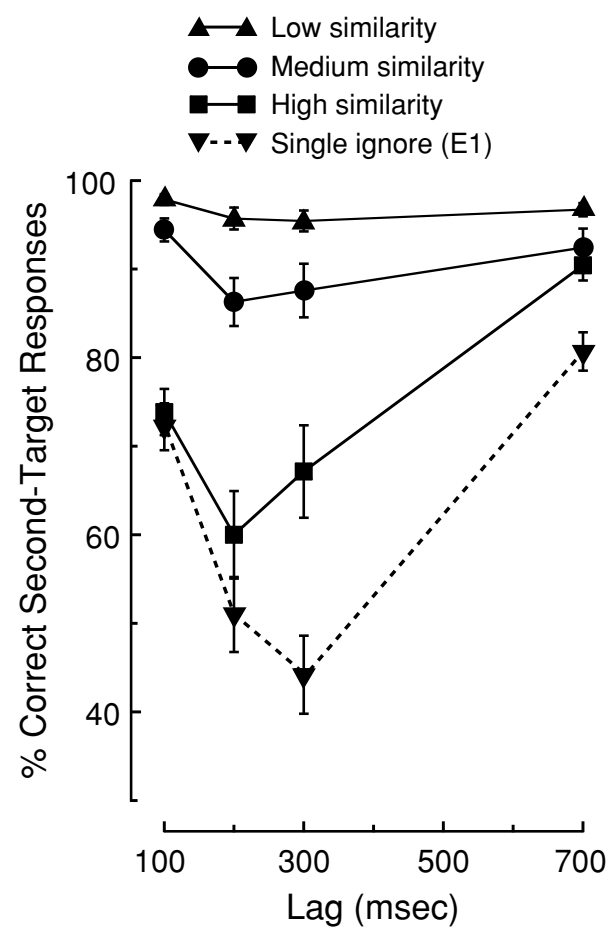

Figure 2. Mean percentages of correct responses in Experiment 2 . The degree of similarity between the second target and the notional first target was highest in the single-ignore condition (E1, Experiment 1), and decreased progressively through the high-, medium-, and low-similarity conditions. Error bars represent one standard error of the mean.

tion in which the first target is to be ignored are based on common — or, at least, shared - mechanisms.

The direct relationship between target-distractor similarity and the magnitude of second-target deficit in Figure 2 is underscored by a comparison between the results of the present experiment and the single-ignore condition in Experiment 1 (the dotted function in Figure 2). The level of target-distractor similarity in the single-ignore condition was higher than that in the high-similarity condition. This is because, being a white letter, the second target in the single-ignore condition shared an additional attribute (color) with the white digit distractors, thus increasing the overall target-distractor similarity beyond that in the highsimilarity condition. In accordance with the hypothesized direct relationship between target-distractor similarity and the magnitude of the second-target deficit, the four functions in Figure 2 are ordered from highest to lowest, as the degree of target-distractor similarity is increased.

Graded effects of target-distractor similarity. The graded effect of target-distractor similarity on the secondtarget deficit illustrated in Figure 2 is consistent with expectations based on the input-filtering hypothesis. Given that the second target was the only item to be reported, it is plausible that the input filter was set optimally for the attributes of the second target. Thus, the probability of a distractor - notably, the first target — passing the filter in- 
creased with its similarity to the second target and, hence, its fit to the filter setting. The increased probability of a distractor passing the filter led to a higher likelihood of the second processing stage being busy when the second target arrived. This resulted in a corresponding increment in the probability of a second-target deficit.

Further support for the input-filtering model is provided by a comparison between the results of the low-similarity and the single-absent conditions. The main difference between them was the color of the second target: white in the single-absent condition and red in the low-similarity condition. The digit distractors were white in both conditions. The important consideration here is that the second target, and hence the setting of the input filter, matched the color of the distractors in the single-absent condition but not in the low-similarity condition. Because of the better match to the setting of the input filter, the probability of a distractor gaining access to the second processing stage was greater in the single-absent than in the low-similarity condition. According to these considerations, accuracy of secondtarget identification should be higher in the low-similarity condition than in the single-absent condition. This was confirmed in a subsidiary 2 between-subjects (condition: single absent and low similarity) $\times 4$ within-subjects (lag: 100, 200, 300, and $700 \mathrm{msec}$ ) ANOVA, which yielded a significant effect of condition $\left[F(1,24)=22.08, M S_{\mathrm{e}}=\right.$ $70.52, p<.001]$. Neither the effect of lag nor the interaction was significant ( $p>.05$ in both cases).

Input filtering and contingent capture. The statistical analysis of the low-similarity and single-absent conditions confirms the graphical evidence in Figures 1 and 2 that the functions for both conditions were essentially flat and parallel throughout the domain. This is in agreement with predictions from the input-filtering model: Any distractor that might have passed the filter would have done so with equal probability at any given lag relative to the second target. This would result in a flat function across lags. Furthermore, because target-distractor similarity was greater in the single-absent condition, the overall accuracy of second-target identification would be expected to be lower in the single-absent than in the low-similarity condition.

The overall pattern of results in Figure 2 is also consistent with expectations based on the phenomenon of contingent capture, in which target identification is impaired if a leading distractor shares the target's defining characteristic (Folk et al., 1992; Ghorashi et al., 2003). As in conventional contingent capture experiments, the participants in the present study were set to identify a single target, and performance suffered when a distractor shared the target's defining attributes. In addition, the results of the present study are consistent with an implicit prediction of contingent capture that, to our knowledge, has not been verified, to date. Implicit in the definition of the factors underlying contingent capture-notably, target-distractor similarity - is the prediction that the magnitude of contingent capture should increase with the similarity between the target and the distractors. Precisely such a relationship is illustrated in Figure 2, underscoring the parallel between contingent capture and the type of second-target deficit that is the hallmark of the AB.

\section{EXPERIMENT 3}

Experiment 3 was designed to address a possible ambiguity in the interpretation of the results of Experiment 1. On the strength of the evidence in Experiment 1, we asserted that using the single-ignore condition in the subtraction procedure resulted in an underestimation of the $\mathrm{AB}$ deficit, and concluded that the single-absent condition was a more appropriate control. That conclusion, however, may be questioned on the grounds that the single-ignore and the single-absent conditions may have differed, not only in respect to the requirement to process the first target, but also in respect to the preparatory set adopted by the observers.

Observers in the single-ignore condition were required to deal with two targets and, therefore, may have adopted a preparatory set that differed from that of observers in the single-absent condition who were required to deal with only one target. It could be argued that a single-target preparatory set might lead to higher accuracy of target identification as compared with a dual-target preparatory set (see, e.g., Bourke, Duncan, \& Nimmo-Smith, 1996). On this option, the difference between the single-absent and either the single-ignore or the dual conditions might represent not only the effect of first-target processing but also the effect of different preparatory sets. To the extent that preparatory set contributes to these differences, the subtraction procedure would overestimate the role of firsttarget processing in determining the magnitude of the $\mathrm{AB}$ deficit.

In Experiment 3, single-absent trials were randomly mixed with dual trials. Observers were told to look for two targets on every trial, and to report both, guessing if unsure. By this procedure, it was assumed that all observers would adopt a dual-task strategy, thus eliminating preparatory set as a possible source of confounding.

\section{Method}

Apparatus, stimuli, and procedures in Experiment 3 were the same as in Experiment 1, except for the following. Fifteen new observers participated in the experiment, which consisted of a single condition that combined the single-absent and the dual conditions of Experiment 1 . Single-absent trials were mixed randomly with dual trials. Participants were told that all trials contained two targets, and were instructed to report both targets, guessing if unsure.

\section{Results and Discussion}

The mean results are illustrated by the solid-line functions in Figure 3, separately for the single-absent and the dual conditions. The corresponding results from Experiment 1 have been added to Figure 3 (dotted-line functions) for ease of comparison. A 2 (Experiments 1 and 3) $\times 4$ (lag: 100, 200, 300, and $700 \mathrm{msec}$ ) ANOVA conducted on the results of the single-absent conditions revealed no significant effects (all $F \mathrm{~s}<1.19$; all $p \mathrm{~s}>.32$ ). A corresponding ANOVA conducted on the dual conditions revealed a significant effect of lag $\left[F(3,75)=83.28, M S_{\mathrm{e}}=\right.$ 


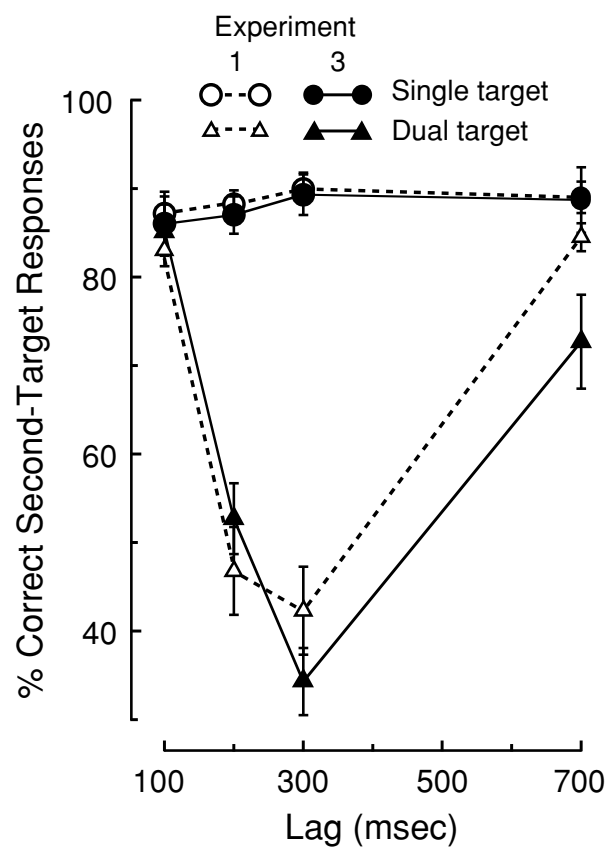

Figure 3. Mean percentages of correct responses in Experiment 3 (solid-line functions). The single-target condition corresponds to the single-absent condition in Experiment 1, and the dual-target condition corresponds to the dual (not-ordered) condition in Experiment 1. The single- and dual-target conditions were mixed randomly across trials. The results of Experiment 1 have been added for ease of comparison (dotted-line functions). Error bars represent one standard error of the mean.

$157.38, p<.001]$ and a significant experiment $\times$ lag interaction $\left[F(3,75)=2.96, M S_{\mathrm{e}}=157.38, p=.038\right]$. The effect of experiment was not significant $(F<1)$.

The results in Figure 3, combined with the outcome of the statistical analyses, clearly indicate that the accuracy of target identification in the single-absent conditions was the same in Experiment 3 as in Experiment 1. From this, it can be concluded that the high level of target identification in the single-absent condition in Experiment 1 was not due to a single-task preparatory set. Had a dual-task preparatory set interfered with target identification in the single-ignore condition in Experiment 1, that interference should have been in evidence as a lower level of performance in the single-absent condition in Experiment 3, relative to the corresponding condition in Experiment 1. From this, we conclude that the use of the single-absent condition in the subtraction procedure does not lead to an overestimate of the $\mathrm{AB}$ deficit and, therefore, is an appropriate control for the effect of the items in the RSVP stream on accuracy of second-target identification.

The results of the dual conditions, although not directly relevant to the principal objective of Experiment 3, are less straightforward. The significant effect of lag was expected. What was more surprising was the significant interaction between lag and experiment. As seen in Figure 3, accuracy of target identification was about the same across the two experiments at the shorter lags, but differed at the longest lag, with performance being somewhat worse in Experiment 3. This difference might well arise from differences in preparatory set. However, it is not clear why such a difference should be in evidence only in the dual condition, and then only at the longer lags. Clearly, this finding requires further investigation.

\section{EXPERIMENT 4}

If the second-target deficit is to be ascribed unambiguously to the consequences of processing the first target, all extraneous sources of interference with second-target processing must be eliminated or controlled. One such extraneous source - the presence of a color singleton - might have been at work in Experiment 2. In that experiment, the magnitude of the second-target deficit was found to be directly related to the similarity between the notional first target and the second target, inviting an explanation based on contingent capture.

It must be realized, however, that in the medium- and high-similarity conditions of Experiment 2, the notional first target might have captured attention not only because it shared a defining attribute with the second target (both red), but because of its salience relative to the other distractors in the RSVP stream (red vs. white). It is known that a salient distractor, even though task irrelevant, can capture attention independently of target-distractor similarity (Theeuwes \& Burger, 1998). Thus, the notional first target in Experiment 2 may have given rise to two independent sources of attentional capture: one based on endogenous goal setting (contingent capture mediated by similarity) and the other based on exogenous capture (mediated by the salient singleton). In either case, attention was captured by the notional first target, to the detriment of the second target. These endogenous and exogenous sources of attentional capture were confounded in Experiment 2.

Experiment 4 was designed to decouple these sources of attentional capture. Exogenous capture by a singleton was assessed by presenting a cluster of green dots in place of the notional first target in the low-similarity condition of Experiment 2, and by comparing the two conditions. The important point is that the green dot singleton did not share a defining feature with the second target (a red letter) and, therefore, would not be expected to mediate contingent capture. The effect of endogenously driven contingent capture was assessed by comparing the green dots condition with a condition in which the cluster of green dots was replaced by a green letter. The green letter was itself a singleton but, by virtue of being a letter, it shared a defining characteristic with the second target and, therefore, would be expected to mediate contingent capture.

Differences in second-target accuracy between the green dots condition and the low-similarity condition of Experiment 2 (in which all elements in the RSVP stream were white except for the second target, which was red) would index the effect of exogenous capture by a singleton. Likewise, differences between the green dots condi- 
tion and the green letter condition would index the effect of contingent capture, independent of exogenous factors.

\section{Method}

Apparatus, stimuli, and procedures in Experiment 4 were the same as in the low-similarity condition of Experiment 2, except for the following. Thirty new observers were allocated randomly to two conditions of 15 observers each. In the green dots condition, the RSVP displays were the same as in the low-similarity condition of Experiment 2, except that the white digit in the notional first-target location was replaced by 30 green dots, distributed randomly within an imaginary square of $13 \times 13$ pixels, which approximated the size of one uppercase alphabetical character. The green letter condition was the same as the green dots condition, except that the cluster of dots was replaced by a green letter.

\section{Results and Discussion}

The mean results are illustrated by the solid-line functions in Figure 4, separately for the green dots and the green letter conditions. The results of the low-similarity condition from Experiment 2 have been added to Figure 4 (dotted-line functions) for ease of comparison.

The effect of a singleton that does not share a defining attribute with the second target was assessed by comparing the results of the green dots condition with those of the low-similarity condition from Experiment 2. The two conditions were identical except for the notional first target, which was a cluster of green dots in one and a white digit in the other. A 2 (condition: low similarity and green dots) $\times 4$ (lag: 100, 200,300, and $700 \mathrm{msec}$ ) ANOVA revealed significant effects of condition $[F(1,27)=6.59$, $\left.M S_{\mathrm{e}}=134.45, p=.016\right]$ and $\operatorname{lag}\left[F(3,81)=4.86, M S_{\mathrm{e}}=\right.$ $19.23, p=.004]$ and a significant interaction $[F(3,81)=$ $\left.8.29, M S_{\mathrm{e}}=19.23, p<.001\right]$.

The graphical evidence in Figure 4, coupled with the results of the statistical analyses, indicate that the presence of a singleton unrelated to the second target produces a lag-dependent second-target deficit indistinguishable from a conventional $\mathrm{AB}$ deficit. This is consistent with the idea that exogenous attentional capture can occur even when the system's endogenous control settings are configured optimally for a different stimulus characteristic (Theeuwes \& Burger, 1998).

This is not to say that similarity does not also play a role. Considered on its own, the green letter condition revealed a significant $\mathrm{AB}$ deficit $\left[F(3,42)=14.02, M S_{\mathrm{e}}=88.31\right.$, $p<.001$ ], consistent with the results of Experiment 2, where similarity was shown to be an important factor. The effect of similarity independent of singleton salience is revealed by comparing the green letter and the green dots conditions. These conditions were identical except that the notional first target shared a defining characteristic of the second target in the former but not in the latter. A 2 (condition: green letter and green dots) $\times 4$ (lag: 100,200 , 300 , and $700 \mathrm{msec}$ ) ANOVA revealed significant effects of condition $\left[F(1,28)=9.71, M S_{\mathrm{e}}=503.83, p=.004\right]$ and lag $\left[F(3,84)=21.50, M S_{\mathrm{e}}=59.08, p<.001\right]$ and a significant interaction $\left[F(3,84)=3.58, M S_{\mathrm{e}}=59.08, p=\right.$ $.017]$. The finding that accuracy of second-target identification was lower in the green letter than in the green dots

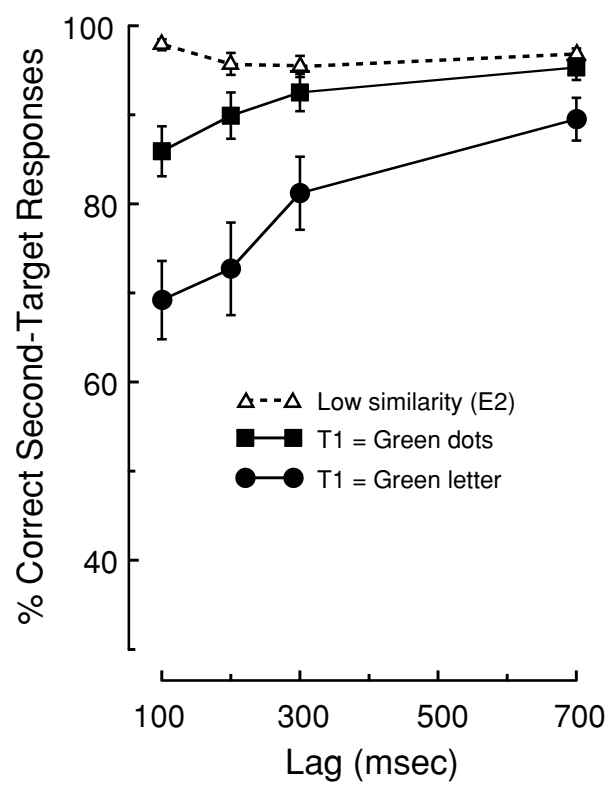

Figure 4. Mean percentages of correct responses in Experiment 4 (solid-line functions). The notional first target (T1) consisted of a cluster of green dots (filled squares) or of a green letter (filled circles). The second target was always a red letter. The results of the low-similarity condition from Experiment 2 (E2) have been added for ease of comparison (dotted-line function). Error bars represent one standard error of the mean.

condition (Figure 4) strongly suggests that endogenous factors influence performance independently of - and in addition to- exogenous factors.

In brief, the results of Experiment 4 point to the importance of both exogenous and endogenous factors in the $\mathrm{AB}$ deficit. The lag-dependent deficit seen in the green dots condition (Figure 4) represents the effect of exogenous factors, and is consistent with Theeuwes and Burger's (1998) claim that attentional capture can be driven exogenously by stimulus salience. By the same token, the lower performance in the green letter relative to the green dots condition is consistent with the claim that attentional capture is driven endogenously by the task's requirements as perceived by the observer (Folk et al., 1992). In light of the present findings, it is clear that the similarity effects observed in Experiment 2 represented the combined actions of endogenous and exogenous factors.

\section{GENERAL DISCUSSION}

We pursued two major objectives in the present work. First, we examined the adequacy of a control procedure, often used in $\mathrm{AB}$ experiments, in which the participants are required to ignore the first of two targets inserted in an RSVP stream of distractors. We proposed that the very act of rejecting the first target involves at least some degree of processing that might interfere with processing of the second target, thereby compromising the validity of the control procedure. 
In Experiment 1, we found that when the observers were required to ignore the first target (single-ignore condition), accuracy of second-target identification was impaired, and followed the time course typically found in $\mathrm{AB}$ experiments. In contrast, when the first target was replaced by a distractor (single-absent condition), so that it acted as a mere placeholder in the RSVP stream, no such impairment was in evidence. Experiment 2 showed that the magnitude of that impairment was directly related to the similarity between the to-be-ignored first target and the to-be-reported second target. This parallels the relationship between target-distractor similarity and the magnitude of $\mathrm{AB}$ deficit typically found in dual-task $\mathrm{AB}$ studies in which observers are required to report both targets (Chun \& Potter, 1995; Dux \& Coltheart, 2005; Maki et al., 1997). We concluded that the second-target deficit found in the conventional control procedure and in dualtask $\mathrm{AB}$ experiments belong to the same class of events. Experiment 3 showed that the difference between the single-ignore and the single-absent conditions in Experiment 1 was not due to different preparatory states.

\section{On the Relationship Between the AB and Attentional Capture}

The second objective of the present work was to examine a parallel between the deficits in target identification obtained in contingent capture and in the $\mathrm{AB}$ paradigms. Because observers are required to report only a single target, the contingent capture paradigm most closely resembles the single-task control conditions studied in Experiments 1 through 3 in the present work. The results of Experiment 4, however, strongly suggest that the scope of such a parallel must be widened. A second-target deficit was obtained in Experiment 4, triggered by a leading singleton that did not share a defining characteristic of the second target and could not, therefore, be expected to mediate contingent capture.

This is not to say that the parallel between the $\mathrm{AB}$ and contingent capture must be abandoned: Rather, it must be expanded to include the more general concept of attentional capture. In attentional capture, processing resources are drawn away from the target in at least two ways. One is when a distractor shares a defining characteristic with the target (i.e., endogenous contingent capture); the other is when a distractor is salient and captures attention even if it does not share any defining characteristic with the target (i.e., exogenous capture by a singleton). In this sense, contingent capture may be regarded as a special case of the more general concept of attentional capture. In any given experiment, either or both forms of capture may have an impact on performance to an extent that depends on the physical characteristics of the display (e.g., the presence of a singleton) and on the observer's attentional set.

\section{A Hybrid Model}

The input-filtering model described previously can handle endogenous capture (i.e., contingent capture) but not the type of exogenous capture mediated by a singleton. Conversely, models based entirely on exogenous fac- tors (e.g., Theeuwes \& Burger, 1998) cannot account for attentional capture that occurs when a distractor is not a salient singleton but shares the target's defining characteristic, as in the single-ignore condition of Experiment 1. What seems to be required is an account that incorporates both endogenous and exogenous sources of attentional capture. Such an account can be given by supplementing the input-filtering model with a hard-wired mechanism that responds automatically to the presence of a salient stimulus, and sends the signal directly to higher processing stages, bypassing the input filter.

In such a hybrid model, an AB deficit would be in evidence whenever attention is diverted from the second target. This could occur either when a singleton is present in the RSVP stream or when a distractor shares a defining feature with the second target. Such a model can account for the results obtained in the present work and for the pattern of results described in the introduction. More cogently, the hybrid model can account readily for the results of Experiment 4, in which the accuracy of second-target identification was higher in the green dots than in the green letter condition. This result is explained on the basis of differences in the probability of the distractor gaining access to higher-level processing, thereby delaying the processing of the second target. In the green letter condition, the distractor could gain access to higher level processing either by passing through the input filter, because it belonged to the same category as the target (both letters), or by bypassing the input filter, because it was a color singleton. In contrast, not sharing a defining feature with the target, the singleton distractor in the green dots condition could gain access to higher-level processing only by bypassing the input filter. Thus, the overall probability of a processing delay for the second target was higher in the green letter than in the green dots condition, with correspondingly higher second-target deficit.

\section{What Control Conditions Should Be Used in AB Experiments?}

The preceding discussion makes it clear that the choice of control condition depends on the issue under study. From a general standpoint, an appropriate control condition for singling out the effect of first-target processing on second-target accuracy is one in which the display sequence is the same as in the experimental condition except for the requirement to process the first target. Such a control is exemplified by the single-absent condition in the present Experiment 1. This control, however, does not permit a separate assessment of endogenous and exogenous factors. If we want to apportion the $\mathrm{AB}$ variance separately to endogenous and exogenous factors, separate control conditions along the lines of those in the present Experiment 4 need to be used.

In this respect, it is worth noting that investigations of the $A B$ have thus far focused invariably on endogenous manipulations, without concern for the possible effects of exogenous factors. Indeed, to our knowledge, the present Experiment 4 is the first instance of exogenous factors being studied separately from endogenous factors. To 
be sure, ostensibly exogenous stimulus attributes such as color and intensity have been included in earlier investigations, but only as a means for implementing endogenous manipulations (see, e.g., Chun, 1997; Seiffert \& Di Lollo, 1997). It goes without saying that the conclusions drawn from these earlier studies are necessarily ambiguous as to the source of the second-target deficit. The theoretical inferences that can be drawn from these studies are, therefore, similarly constrained.

\section{Concluding Comments}

From a purely pragmatic standpoint, the present work addresses the issue of control procedures in the AB, an issue that hitherto had not been addressed explicitly. In pursuing this objective, we arrived at a distinction between endogenous and exogenous factors that contribute to the $\mathrm{AB}$ and that require distinct control conditions for their assessment. From a theoretical standpoint, the distinction between endogenous and exogenous factors prompted the development of a hybrid input-filtering model of the AB. In that model, the second-target deficit occurs when a stimulus presented prior to the second target gains access to a higher processing stage, thereby causing a delay in the processing of the second target. This can occur when a leading stimulus passes the input filter, because it shares a defining attribute of the target or, in the case of a singleton, when it bypasses the input filter via a hardwired pathway.

In either case, a parallel can be established between the AB literature and the literature on attentional capture, which shows that capture can occur endogenously (contingent capture) or exogenously (via singleton). This parallel is reinforced by the finding that the target-identification deficit follows similar time courses in the $\mathrm{AB}$ and in the attentional capture paradigms (Ghorashi et al., 2003). The clear advantage of bringing the $\mathrm{AB}$ and attentional capture within a single conceptual rubric is that the outcomes obtained with two ostensibly distinct paradigms can be explained on the basis of the same underlying mechanisms.

\section{REFERENCES}

Arnell, K. M., \& Joliceur, P. (1999). The attentional blink across stimulus modalities: Evidence for central processing limitations. Journal of Experimental Psychology: Human Perception \& Performance, 25, 630-648.

Bourke, P. A., DuncAN, J., \& Nimmo-SMith, I. (1996). A general factor involved in dual-task performance decrement. Quarterly Journal of Experimental Psychology, 49A, 525-545.

Broadbent, D. E., \& Broadbent, M. H. (1987). From detection to identification: Response to multiple targets in rapid serial visual presentation. Perception \& Psychophysics, 42, 105-113.

Christmann, C., \& Leuthold, H. (2004). The attentional blink is susceptible to concurrent perceptual processing demands. Quarterly Journal of Experimental Psychology, 57A, 357-377.

Chun, M. M. (1997). Temporal binding errors are redistributed by the attentional blink. Perception \& Psychophysics, 59, 1191-1199.

Chun, M. M., \& PotTer, M. C. (1995). A two-stage model for multiple target detection in rapid serial visual presentation. Journal of Experimental Psychology: Human Perception \& Performance, 21, 109-127.
Di Lollo, V. (1980). Temporal integration in visual memory. Journal of Experimental Psychology: General, 109, 75-97.

Duncan, J. (1980). The locus of interference in the perception of simultaneous stimuli. Psychological Review, 87, 272-300.

Dux, P. E., \& Coltheart, V. (2005). The meaning of the mask matters: Evidence of conceptual interference in the attentional blink. Psychological Science, 16, 775-779.

Egeth, H. E., Folk, C. L., Leber, A. B., Nakama, T., \& Hendel, S. K. (2001). Attentional capture in the spatial and temporal domains. In C. L. Folk and B. S. Gibson (Eds.), Attraction, distraction and action: Multiple perspectives on attentional capture (pp. 93-119). New York: Elsevier.

Folk, C. L., Remington, R. W., \& Johnston, J. C. (1992). Involuntary covert orienting is contingent on attentional control settings. Journal of Experimental Psychology: Human Perception \& Performance, 18, 1030-1044.

Ghorashi, S. M. S., Zuvic, S. M., Visser, T. A. W., \& Di Lollo, V. (2003). Focal distraction: Spatial shifts of attentional focus are not required for contingent capture. Journal of Experimental Psychology: Human Perception \& Performance, 29, 78-91.

Giesbrecht, B., \& Di Lollo, V. (1998). Beyond the attentional blink: Visual masking by object substitution. Journal of Experimental Psychology: Human Perception \& Performance, 24, 1454-1466.

Hoffman, J. E. (1979). A two-stage model of visual search. Perception \& Psychophysics, 25, 319-327.

Jolicceur, P., \& Dell'AcQua, R. (1998). The demonstration of shortterm consolidation. Cognitive Psychology, 36, 138-202.

Kellie, F. J., \& Shapiro, K. L. (2004). Object file continuity predicts attentional blink magnitude. Perception \& Psychophysics, 66, 692712.

Maki, W. S., Bussard, G., Lopez, K., \& Digby, B. (2003). Sources of interference in the attentional blink: Target-distractor similarity revisited. Perception \& Psychophysics, 65, 188-201.

Maki, W. S., Couture, T., Frigen, K., \& Lien, D. (1997). Sources of the attentional blink during rapid serial visual presentation: Perceptual interference and retrieval competition. Journal of Experimental Psychology: Human Perception \& Performance, 23, 1393-1411.

Potter, M. C., Chun, M. M., Banks, B. S., \& Muckenhoupt, M. (1998). Two attentional deficits in serial target search: The visual attentional blink and an amodal task-switch deficit. Journal of Experimental Psychology: Learning, Memory, \& Cognition, 24, 979-992.

Raymond, J. E., Shapiro, K. L., \& Arnell, K. M. (1992). Temporary suppression of visual processing in an RSVP task: An attentional blink? Journal of Experimental Psychology: Human Perception \& Performance, 18, 849-860.

Seiffert, A. E., \& Di Lollo, V. (1997). Low-level masking in the attentional blink. Journal of Experimental Psychology: Human Perception \& Performance, 23, 1061-1073.

Shapiro, K. L., Raymond, J. E., \& Arnell, K. M. (1994). Attention to visual pattern information produces the attentional blink in rapid serial visual presentation. Journal of Experimental Psychology: Human Perception \& Performance, 20, 357-371.

Theeuwes, J., \& Burger, R. (1998). Attentional control during visual search: The effect of irrelevant singletons. Journal of Experimental Psychology: Human Perception \& Performance, 24, 1342-1353.

Visser, T. A. W., Bischof, W. F., \& Di Lollo, V. (1999). Attentional switching in spatial and nonspatial domains: Evidence from the attentional blink. Psychological Bulletin, 125, 458-469.

Visser, T. A. W., Bischof, W. F., \& Di Lollo, V. (2004). Rapid serial visual distraction: Task-irrelevant items can produce an attentional blink. Perception \& Psychophysics, 66, 1418-1432.

WARD, R., Duncan, J., \& ShapIro, K. (1997). Effects of similarity, difficulty, and nontarget presentation on the time course of visual attention. Perception \& Psychophysics, 59, 593-600.

(Manuscript received February 4, 2005; revision accepted for publication July 25, 2005.) 